\title{
Spontaneous mutations in a regulatory gene induce phenotypic heterogeneity and adaptation of Ralstonia solanacearum to changing environments
}

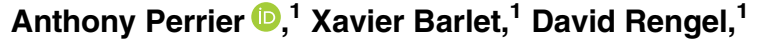 \\ Philippe Prior, ${ }^{2}$ Stéphane Poussier, ${ }^{3}$ Stéphane Genin ${ }^{1}$ \\ and Alice Guidot $\mathbb{B}^{1^{*}}$ \\ ${ }^{1}$ LIPM, Université de Toulouse, INRA, CNRS, Castanet- \\ Tolosan, France. \\ ${ }^{2} U M R$, Peuplements Végétaux et Bioagresseurs en \\ Milieu Tropical, INRA, Saint-Pierre, Réunion, France. \\ ${ }^{3} U M R$, Peuplements Végétaux et Bioagresseurs en \\ Milieu Tropical, Université de la Réunion, Saint-Pierre, \\ Réunion, France.
}

\section{Summary}

An evolution experiment with the bacterial plant pathogen Ralstonia solanacearum revealed that several adaptive mutations conferring enhanced fitness in plants arose in the efp $R$ gene encoding a regulator of virulence and metabolic functions. In this study, we found that an efpR mutant systematically displays colonies with two morphotypes: the type S ('smooth', similar to the wild type) and the type EV ('efpR variant'). We demonstrated that the efpH gene, a homologue of efpR, plays a key role in the control of phenotypic heterogeneity, the $\Delta$ efpR- $\Delta$ efpH double mutant being stably locked into the EV type. Using mixed infection assays, we demonstrated that the type EV is metabolically more proficient than the type $S$ and displays fitness gain in specific environments, whereas the type $S$ has a better fitness into the plant environment. We provide evidence that this efpR-dependent phenotypic heterogeneity is a general feature of strains of the $R$. solanacearum species complex and could occur in natural conditions. This study highlights the potential role of phenotypic heterogeneity in this plant pathogen as an adaptive trait to changing environments.

Received 28 January, 2019; revised 11 June, 2019; accepted 13 June, 2019. *For correspondence. E-mail alice.guidot@ inra.fr; Tel. (+33) 05612855 92; Fax (+33) 05612850 61. This paper is dedicated to the memory of our wonderful friend and colleague, Philippe Prior, who passed away on November 11, 2018.

\section{Introduction}

In bacterial clonal populations, individual cells often harbour variation in their phenotype even when living in the same environment. The most common observation is variation in the morphology of colonies arising from single clones (Workentine et al., 2013; Ronin et al., 2017), but other phenotypic variations are also routinely observed such as antibiotic resistance, motility, growth profiles or biofilm formation (Chai et al., 2008; Workentine et al., 2013; Arnoldini et al., 2014). The molecular mechanisms that lead to phenotypic heterogeneity in bacteria are very diverse and include phase variation, gene expression noise, post-transcriptional modification or epigenetic mechanisms (Hallet, 2001; Acar et al., 2008; Veening et al., 2008; Fraser and Kaern, 2009; Casadesús and Low, 2013). Phenotypic heterogeneity has been widely reported for bacterial pathogens with the description of co-existing virulent and avirulent (or less virulent) subpopulations (Diard et al., 2013; Mouammine et al., 2017; Ronin et al., 2017; Chin et al., 2018).

The development of heterogeneous subpopulations during host infection has been associated with various adaptive strategies (Ackermann, 2015; Weigel and Dersch, 2018). First, phenotypic heterogeneity can allow a genotype to survive in fluctuating environments (Rainey et al., 2011; Schreiber et al., 2016). For example, in Salmonella, heterogeneous expression of virulence factors allows a subset of the population to escape host inflammatory responses (Stewart et al., 2011; Tuchscherr et al., 2011). Another illustration of such bacterial bet-hedging strategy is the formation of 'persister' cells such as those resistant to antibiotics (Balaban et al., 2004). A second adaptive explanation for phenotypic heterogeneity is the cooperation, or 'division of labour', strategy. Here, the phenotypically different sub-populations differentiate and cooperate to survive in non-fluctuating environments (Weigel and Dersch, 2018). Another possibility in isogenic subpopulation relationships involves the cell-to-cell variation in the production of public goods. Here, nonproducing phenotypic variants behave like cheaters, which benefit from the subpopulation of producing cells without paying the metabolic costs (Strassmann, 2000; Harrison, 2013). 
Very little is known about phenotypic heterogeneity of bacterial pathogens in non-mammalian hosts. The first example was reported for the plant pathogen Pseudomonas syringae, for which structural and regulatory components of the type 3 secretion system were shown to display heterogeneous expression within plant tissues (Rufián et al., 2016, 2018). This bistable pattern of gene expression correlates with differences in virulence and is dependent upon complex regulatory loops, but the mechanism behind this phenomenon is not yet understood (Rufián et al., 2016).

In an evolution experiment with the bacterial plant pathogen Ralstonia solanacearum (Guidot et al., 2014), we observed the emergence of isogenic clones displaying phenotypic heterogeneity. Ralstonia solanacearum is the agent of bacterial wilt disease on more than 250 plant species around the world, mainly in tropical and sub-tropical countries (Mansfield et al., 2012; Peeters et al., 2013). This soil borne betaproteobacterium infects plants through the roots and invades the xylem vessels where it multiplies extensively, causing wilting symptoms and plant death. To identify determinants involved in host adaptation, experimental evolution of $R$. solanacearum was conducted by serial passage experiments from one plant to another during approximately 300 in planta bacterial generations in order to select for individuals with enhanced fitness (Guidot et al., 2014). Interestingly, genome sequencing of such individuals revealed that single nucleotide polymorphisms (SNPs) appeared in a single gene, named efp $R$, in six independent lineages evolved on different hosts (Guidot et al., 2014; Perrier et al., 2016). efp $R$ is a small gene (342 bp) encoding a transcriptional regulator and all but one SNPs identified were non-synonymous mutations. A reverse genetic approach confirmed that the SNPs detected in the efp $R$ gene explained the fitness gain on plants (Guidot et al., 2014; Perrier et al., 2016). Further studies revealed that EfpR acts as a global catabolic repressor affecting several metabolic pathways; it also controls the expression of important virulence traits such as motility and the production of exopolysaccharides (EPS) (Perrier et al., 2016; Capela et al., 2017) and is therefore connected to the $R$. solanacearum virulence regulatory network (Peyraud et al., 2016; 2018).

Here, we report that a $R$. solanacearum isogenic population carrying a mutation in the efp $R$ gene systematically displays two different morphotypes of colonies on plates, thus revealing a phenotypic heterogeneity phenomenon. We investigated the functional relevance of such biological diversity generated through efp $R$ mutations and its contribution to population dynamics in planta or in other environments. Importantly enough, a survey on $R$. solanacearum isolates from strain collections revealed that phenotypic heterogeneity behaviour can be detected among strains of the $R$. solanacearum species complex.

\section{Results}

Loss of function of efpR generates phenotypic heterogeneity

On complete medium plates supplemented with D-glucose and triphenyl tetrazolium chloride, the $R$. solanacearum wild-type (WT) strain GMl1000 forms fluidal and pink centered colonies (Fig. 1A). We observed that the efp $R$ deleted mutant ( $\Delta$ efpR strain) always produces two types of colonies on this medium: the first type is similar to the WT strain and was referred hereafter as type $S$ (smooth), whereas the second type forming red, less mucoid colonies was designated type EV (efpR variant) (Fig. 1B). After subsequent subcultures, both isolated $\mathrm{EV}$ and $\mathrm{S}$ type colonies again systematically display colonies with the two morphotypes. This phenotypic heterogeneity phenomenon was observed for the $\Delta$ efp $R$ strain but also for all the efpR SNP mutants that were selected during the evolution experiment in planta (Guidot et al., 2014) (Supporting Information Fig. S1).

In a former study, we identified in the $R$. solanacearum genome a homologue of efp $R, R S c 3149$ hereafter named efp $H$, encoding for a putative transcription regulator having $79 \%$ protein identity with EfpR (Perrier et al., 2016). We investigated whether this efpR-homologue also played a role in the advent of phenotypic heterogeneity observed with the $\Delta$ efp $R$ strain. We generated a $\Delta$ efpH deletion strain and this strain had a WT S morphotype when plated on complete medium (Supporting Information Fig. S1). However, the $\Delta$ efp $R-\Delta$ efpH double mutant only formed EV type colonies (Fig. 1C and Supporting Information Fig. S1). The phenotype of the double mutant even appeared more pronounced than the $\Delta$ efpR-EV type with almost non-mucoid colonies at $52 \mathrm{~h}$. However, after $72 \mathrm{~h}$ the efpR-EV colonies and $\Delta$ efpR$\Delta$ efpH colonies looked similar. We never observed the occurrence of $\mathrm{S}$ type colonies from the $\Delta$ efpR- $\Delta$ efpH double mutant strain, suggesting that this strain is 'locked' into the EV morphotype.

\section{$S$ and EV morphotypes of the efpR mutants display specific gene expression patterns}

To investigate the variation of gene expression occurring between the EV and S types, we constructed two transcriptional fusions with the respective promoters of $R S c 3148$ and $\mathrm{fliC}$ and the mCherry red fluorescent protein reporter gene. Both $R S c 3148$ and fliC genes were previously demonstrated to be among the top upregulated genes in the $\Delta$ efpR strain compared to the WT strain (Perrier et al., 2016; Capela et al., 2017). We found that in the $S$ colonies formed by the $\Delta$ efpR mutant both promoters were either not expressed or expressed at a very low level, similarly to what happens in the $\mathrm{S}$ colonies formed by the WT strain 

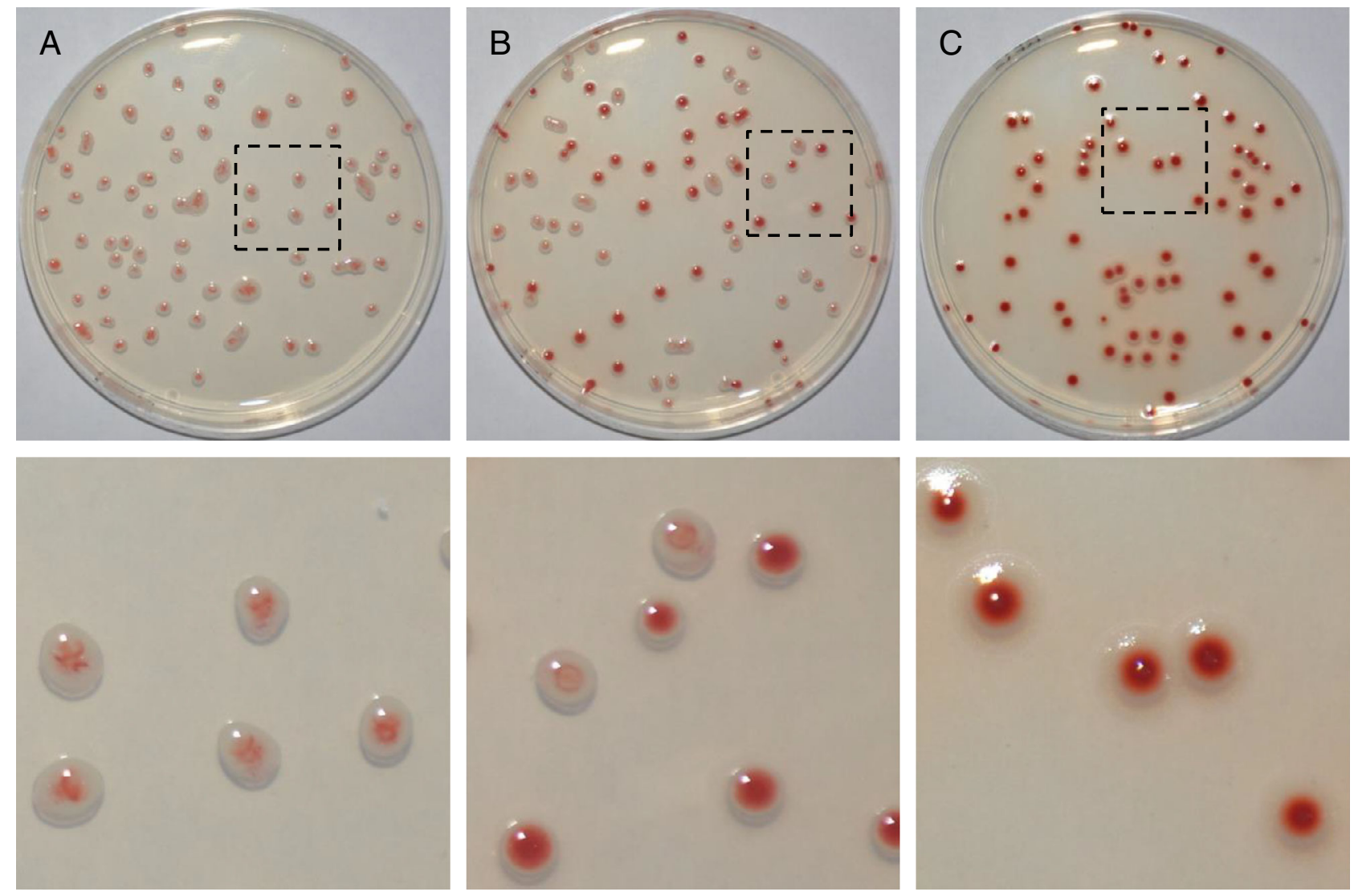

Fig. 1. Ralstonia solanacearum GMl1000 $\Delta$ efpR strain generates phenotypic heterogeneity. Colonies of the WT (A), $\Delta$ efpR (B) and the $\Delta e f p R$ $\Delta e f p H(C)$ strains grown $52 \mathrm{~h}$ on complete medium agar plates supplemented with D-glucose and triphenyl tetrazolium chloride red. A zoom-in view corresponding to the dashed square zone is represented below each top-panel. The WT (S type) forms pink and fluidal colonies whereas the EV type appears as red and less mucoid colonies.

(Fig. 2 and Supporting Information Fig. S2). On the other hand, the RSc3148 and fliC promoters were both strongly expressed in the EV colonies suggesting a specific expression pattern in EV colonies compared to the $\mathrm{S}$ ones (Fig. 2 and Supporting Information Fig. S2). As expected, in the colonies formed by the $\Delta$ efp $R-\Delta$ efpH mutant producing only $\mathrm{EV}$ colonies, the $R S C 3148$ and fliC promoters were strongly expressed in all the colonies (Fig. 2 and Supporting Information Fig. S2). Microscopic observations were also conducted at the cellular level to analyse the activity of the fliC promoter fused to a GFP reporter gene (Supporting Information Fig. S3). The analysis revealed that fliC is weakly expressed in WT strain cells (S type), highly expressed in $\Delta$ efpR$\Delta e f p H$ mutant cells (EV type) while in the efpR mutant ( $\mathrm{S}+\mathrm{EV}$ type), cells were either expressing fliC or not (residual expression).

\section{Transcriptomic profiling confirms that the $\Delta$ efpR- $\Delta \mathrm{efpH}$ double mutant is locked into the EV morphotype}

To obtain a view of the global gene expression pattern in the EV morphotype, we examined the transcriptomic profiles of the $\Delta$ efpR (EV colonies inoculum) and the $\Delta$ efpR- $\Delta$ efpH (EV) strains in comparison with the WT (S) during exponential growth in complete medium $\left(\mathrm{OD}_{600 \mathrm{~nm}} \sim 0.5\right)$ (Supporting

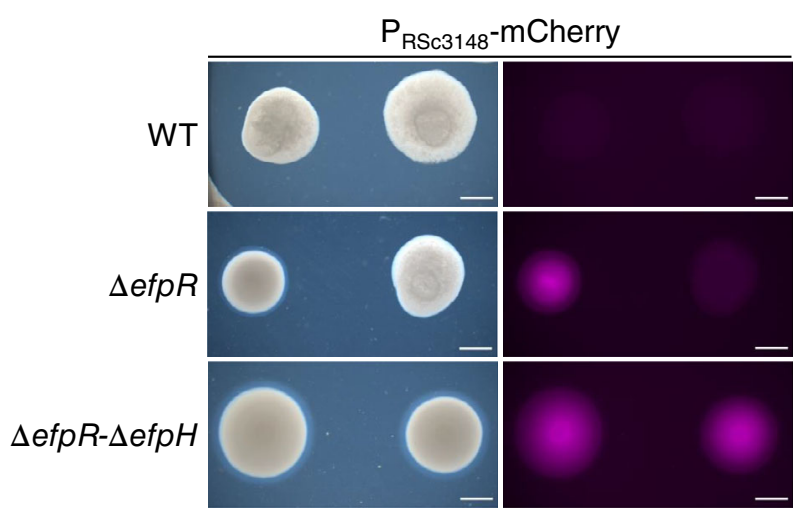

Fig. 2. Differential expression pattern of the RSc3148 promoter in S and EV type colonies. Left panels show bright field images; the right panels show the same colonies under fluorescence excitation. For the $\Delta$ efpR strain, one type EV (left) and one type S (right) colonies are shown. Expression of the $P_{\mathrm{RSc} 3148}$-mCherry reporter occurs only in EV type colonies. Bacteria were grown on complete medium agar plates for $72 \mathrm{~h}$ at $28^{\circ} \mathrm{C}$. Scale bar $=2 \mathrm{~mm}$. 
Information Table S1). We found 674 (13.67\%) differentially expressed genes (DEGs) (>twofold differentially expressed; $p$-value (padj,FDR) $<0.05)$ between the WT strain and $\Delta$ efp $R$ strain, and $553(11.22 \%)$ DEGs between the WT strain and the $\Delta$ efpR- $\Delta$ efpH strain (Supporting Information Table S2). In total, 743 genes were either differentially expressed in the $\Delta$ efpR-EV or the $\Delta$ efpR- $\Delta$ efpH mutants compared to the WT strain, with 484 DEGs shared by both strains, 190 specific to the $\Delta e f p R$ strain and 69 specific to the $\Delta$ efpR- $\Delta$ efpH strain (Supporting Information Table S2). Comparison of the transcriptomes of the $\Delta$ efp $R$-EV strain with the $\Delta$ efpR- $\Delta$ efpH strain revealed only 25 DEGs (i.e. $0.51 \%$ of the expressed genes), indicating that the gene expression pattern was similar in both strains having an EV phenotype (Supporting Information Table S2). Among these 25 DEGs, 18 were downregulated in both strains compared to the WT strain including 12 genes belonging to the EPS biosynthesis cluster, 4 metabolism-related genes and 2 genes coding for a lectin and a putative transmembrane protein respectively. The observation that several EPS biosynthetic genes were more strongly repressed in the double mutant than in the single efp $R$-EV mutant was in agreement with the more pronounced phenotype of the $\Delta \operatorname{efpR-\Delta efpH}$ double mutant on $52 \mathrm{~h}$ plates (less mucoid aspect, see Fig. 1C). Considering that (i) the transcriptomic profiles of the efpR-EV type strain and the $\Delta$ efpR- $\Delta$ efpH double mutant largely overlap and (ii) the $\Delta e f p R-\Delta e f p H$ was never observed to revert to WT phenotype, we concluded that the $\Delta$ efpR- $\Delta$ efpH is 'locked' into the EV morphotype.

\section{The EV morphotype is strongly altered in pathogenicity}

We previously showed that the $\Delta e f p R$ mutant had reduced virulence on susceptible tomato plants compared to the WT

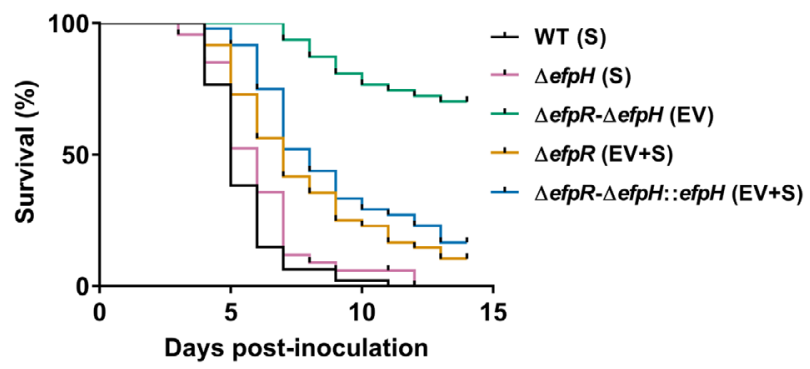

Fig. 3. Virulence of the EV type is strongly impaired on tomato. Kaplan-Meier survival analysis of tomato plants inoculated with the WT strain, the $\Delta$ efp $R$ and the $\Delta$ efpH single mutants, the $\Delta$ efpR$\Delta$ efpH double mutant and the $\Delta$ efpR- $\Delta$ efpH $:: \Delta$ efpH complemented strain. The variant type (S or/and EV) found in the different genetic background is indicated in brackets. Curves represent three independent biological repeats of $15-16$ plants inoculated by soil drenching. All the survival curves are significantly different from the WT survival curve (Gehan-Breslow-Wilcoxon test; $p<0.0001$ ) except the survival curve of the $\Delta$ efpH single mutant (GehanBreslow-Wilcoxon test; $p=0.0819$ ). strain (Perrier et al., 2016). Because the $\Delta$ efpR strain is phenotypically heterogeneous, we wanted to assess more accurately the respective contribution of the $E V$ and $S$ types to pathogenicity. We took advantage of the $\Delta$ efpR- $\Delta$ efpH double mutant as a stable EV type to evaluate its virulence on tomato plants. This double mutant was compared to the WT (type S), the $\Delta$ efpH strain (type S), the $\Delta$ efpR (type $\mathrm{S}+\mathrm{EV})$ strain, and the $\Delta$ efpR- $\Delta$ efpH double mutant complemented with the efpH gene (type S + EV) (Fig. 3 and Supporting Information Fig. S1). The Kaplan-Meier survival analysis of tomato plants showed a significantly better survival for plants inoculated with all mutants compared to the WT strain, except for the $\Delta$ efpH mutant (Gehan-BreslowWilcoxon test; $p<0.0001$ ), indicating that the presence of EV cells tends to decrease the disease symptom rate. The survival curve observed with the $\Delta$ efpR- $\Delta$ efpH strain showed that this double mutant was strongly altered for pathogenicity and significantly more than the $\Delta$ efpR strain (Gehan-Breslow-Wilcoxon test; $p<0.0001$ ). On the other hand, the behaviour of the complemented strain $(\Delta e f p R-$ $\Delta$ efpH::efpH) was not statistically different from the $\Delta$ efpR strain, indicating that the presence of a functional efpH gene was able to restore phenotypic heterogeneity and so to increase the disease symptom rate on tomato (Fig. 3).

Fitness of the EV type is reduced in planta but increases in the presence of the S type

We compared the dynamic behaviour of the EV and S subpopulations in planta by conducting mixed infection assays of tomato plant stems. Because the $\Delta$ efpR mutant generates both $E V$ and $S$ types, in order to control the ratio of the EV and $S$ types in our inoculums, we conducted these assays with the WT strain as the S type and the $\Delta$ efpR- $\Delta$ efpH strain as the EV type. Bacterial populations were recovered from stem sections 3 days after stem injection and the relative proportion of each strain quantified by GFP and mCherry fluorescent markers. This experiment first showed that the colonization of a homogeneous EV (EV+[EV]) population $\left(3.6 \pm 3.0 \times 10^{8} \mathrm{CFU} \mathrm{g}^{-1} \mathrm{FW}\right)$ was strongly reduced compared to the homogeneous WT $S(\mathbf{S}+[\mathbf{S}])$ population $\left(1.8 \pm 0.79 \times 10^{9} \mathrm{CFU} \mathrm{g}^{-1} \mathrm{FW} ; p\right.$-value $<0.001$, KruskalWallis test with Dunn's post-test) (Fig. 4). In mixed populations, the $S(\mathbf{S}+[E V])$ type had a significantly reduced colonization $\left(9.0 \pm 5.9 \times 10^{8} \mathrm{CFU} \mathrm{g}^{-1} \mathrm{FW} ; p<0.001\right) \mathrm{com}-$ pared to the homogeneous $\mathbf{S}(\mathbf{S}+[\mathbf{S}])$ type reference, indicating that the EV type had a detrimental effect on the fitness of the $S$ type (Fig. 4). However, monitoring the outcome of the EV subpopulation from a mixed infection with the $S$ type $(\mathrm{EV}+[\mathrm{S}])$ revealed that co-infection was much beneficial for the EV type (reaching $2.5 \pm 1.7 \times 10^{9} \mathrm{CFU} \mathrm{g}^{-1} \mathrm{FW}$, statistically different when compared to the homogeneous $\mathrm{EV}$ $(\mathrm{EV}+[\mathrm{EV}])$ population; $p<0.01)$ (Fig. 4). 
In order to rule out the possibility that the 1:1 initial infection ratio could induce a bias in the observed results, we performed similar co-inoculation experiments by varying the initial ratio of the mixed infection assay (100:0, 99:1, 90:10, 50:50 and 10:90). The obtained results showed that the $S$ type population size from a $\mathbf{S}+[\mathrm{EV}]$ mix started to decrease in the presence of only $1 \%$ of EV population (Supporting Information Fig. S4). It also confirmed that, conversely, the EV population size increased in presence of the $S$ type (EV+[S]) (Supporting Information Fig. S4). We therefore concluded that the EV type is strongly compromised for in planta fitness but takes advantage from the presence of the S type to maximize its in planta growth.

\section{The EV type could benefit in planta from the EPS} produced by the $S$ type

Ralstonia solanacearum secretes massive amounts of EPS in planta, where they act both as a colonization and a virulence factor (Genin and Denny, 2012). The EV type is strongly impaired in its capacity to secrete EPS, and so produces less mucoid colonies than the S type (Fig. 1). We hypothesized that EPS produced and secreted by the S type could be beneficial to the EV type, thus improving its ability for colonization in planta. To test this hypothesis, we introduced into the WT and the $\Delta$ efpR- $\Delta$ efpH double mutant strains a $\mathrm{P}_{\mathrm{TAC}}-\mathrm{xps} R$ construct allowing the constitutive expression of $x p s R$, which is a positive regulator of the EPS biosynthetic genes (Huang et al., 1995), acting independently of efpR. We investigated the virulence of the WT, the $\Delta$ efp $R-\Delta$ efpH and the $x p s R:: \Omega$ strains bearing or not the $\mathrm{P}_{\mathrm{TAC}}-x p s R$ construct on susceptible tomato plants. The

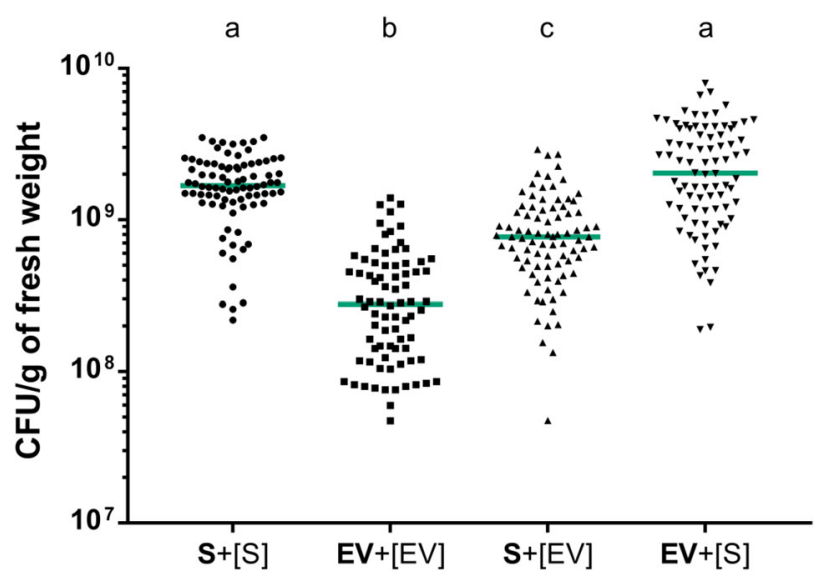

Fig. 4. In planta fitness of the EV and $S$ types during co-infection. Bacteria were co-injected in tomato stems at a $1: 1$ ratio and harvested 3 days post-inoculation. Each dot represents the population size of the strain indicated in bold within a single plant after coinoculation with the strain indicated in brackets. Horizontal bars indicate the median. Three independent biological repeats were performed with 10-18 plants per repeats per conditions. Kruskal-Wallis and Dunn's multiple comparison test were used to establish statistical groups $(p<0.001)$. S $=$ WT strain; $\mathrm{EV}=\Delta$ efpR- $\Delta$ efpH strain. constitutive expression of $x p s R$ in the $\Delta$ efp $R-\Delta$ efp $H$ and the $x p s R:: \Omega$ strains significantly improved their aggressiveness (Gehan-Breslow-Wilcoxon test; $p<0.0001$ ) even if these two strains remained less aggressive than the WT strain (Gehan-Breslow-Wilcoxon test; $p<0.0001$ ) (Fig. 5). These results therefore suggested that EPS produced by the WT (or S type) can rescue the EV type in planta to improve its colonization and virulence.

The EV type shows fitness gain in environments outside the plant

To investigate the behaviour of a mixed population $(E V+S)$ in another environment than in planta, we performed an in vitro competition assay between the S (WT) and EV $(\Delta$ efpR- $\Delta$ efpH) types in complete medium and in minimal medium supplemented with different carbon sources (Fig. 6). Mixed populations with an initial 1:1 ratio between the EV and $S$ types were cultivated in liquid minimal medium with a sole carbon source. The relative proportion of the EV and $S$ types was then quantified when populations were up to $10^{9} \mathrm{CFU} \mathrm{ml}{ }^{-1}$ or above. In minimal medium, we found that the proportion of EV cells reached $75.6 \pm 5.4 \%$ (L-histidine), $88.3 \pm 2.2 \%$ (L-glutamine), $92.9 \pm 2.9 \%$ (L-asparagine) and $96.3 \pm 1.3 \%$ (L-proline), whereas in complete medium, the EV/S type ratio was close to $50 \%(45.3 \pm 6.4 \%$ of EV cells) (Fig. 6).

We then compared the growth dynamic of populations of the $\Delta$ efp $R$ strain in liquid minimal medium + L-proline inoculated with a single colony of either the S or EV type (initial population corresponding to an $\mathrm{OD}_{600 \mathrm{~nm}}$ of 0.05). The culture inoculated with the $\Delta$ efpR-EV type reached a mean $\mathrm{OD}_{600 \mathrm{~nm}}$ of 1.0 after $40.9 \mathrm{~h}$ while it took $61.7 \mathrm{~h}$ to reach the same mean $\mathrm{OD}_{600 \mathrm{~nm}}$ for the one inoculated with the $\Delta$ efpR$S$ type in the same condition (Fig. 7). In comparison, the WT

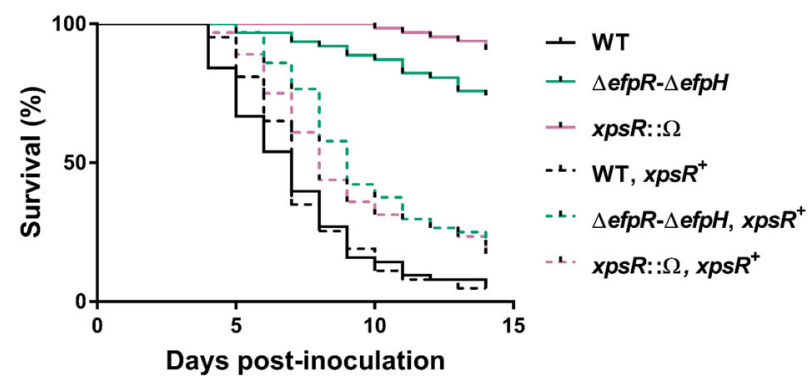

Fig. 5. Virulence of the EV type is partially restored by constitutive expression of $x p s R$, a positive regulator of eps genes. Kaplan-Meier survival analysis of tomato plants inoculated with the WT, the $\Delta \operatorname{efp} R-\Delta \operatorname{efpH}$ and the $x p s R:: \Omega$ strains with and without the $\mathrm{P}_{\mathrm{TAC}^{-}}$ $x p s R$ construct $\left(x p s R^{+}\right)$. Curves represent three independent biological repeats of 15-16 plants inoculated by soil drenching. All the survival curves are significantly different from the WT survival curve (Gehan-Breslow-Wilcoxon test, $p<0.005$ ) except the survival curve of the WT, $x p s R^{+}$strain (Gehan-Breslow-Wilcoxon test; $p=0.3637)$. 


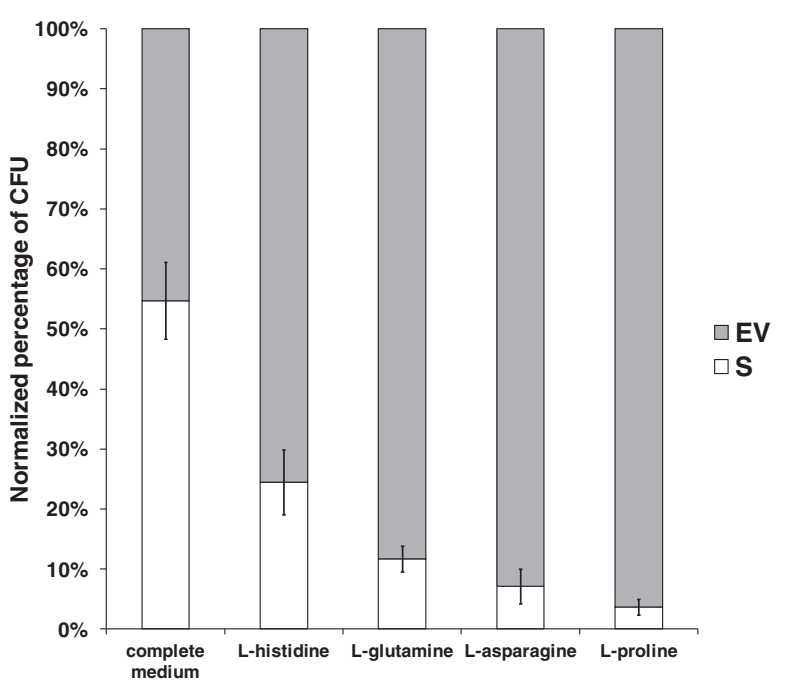

Fig. 6. The EV type provides fitness gain in specific environments. In vitro competition assay between the $\operatorname{EV}(\Delta$ efpR- $\Delta$ efpH $)$ and $\mathrm{S}$ (WT) types inoculated at a 1:1 ratio in either complete medium or minimal medium supplemented with one amino acid as a sole carbon source. Competition experiments started with $\sim 5.10^{5} \mathrm{CFU} \mathrm{ml}^{-1}$ and were stopped when cultures were above $10^{9} \mathrm{CFU} \mathrm{ml}^{-1}$. The percentage of CFU recovered was normalized by the percentage of CFU present in the inoculum. Two independent biological repeats, each comprising three technical repeats, were performed.

strain only reached a mean $\mathrm{OD}_{600 \mathrm{~nm}}$ of 0.55 after $90 \mathrm{~h}$ (Fig. 7). This result confirmed that the growth rate of the EV type in minimal medium+ L-proline is significantly higher than the $S$ type. The finding that growth of the culture inoculated with the $\Delta$ efpR-S type was much better than the WT culture, also suggested that this behaviour was dependent on the phenotypic heterogeneity occurring in the $\Delta \operatorname{efp} R$ genetic background. To ascertain this hypothesis, we determined for each strain the percentage of EV type CFU in the inoculum and at the end of the exponential growth phase. In cultures inoculated with $\Delta$ efpR-EV type colonies, the percentage of EV type CFU was $69.9 \pm 11.3 \%$ in the inoculum and reached $92.3 \pm 3.4 \%$ at the end of the exponential growth phase (Fig. 7). For the cultures inoculated with the $\Delta$ efpR-S type, the percentage of EV type CFU was $1.6 \pm 0.3 \%$ in the inoculum and reached $87.8 \pm 5.0 \%$ at the end of the exponential growth phase. This analysis thus revealed that even a low percentage of EV cells in the $R$. solanacearum population allows the bacterial population to reach a higher cell density in this environment compared to a population exclusively composed of $S$ cells (WT strain).

\section{Phenotypic heterogeneity can be detected among natural $\mathrm{R}$. solanacearum isolates}

The efpR gene was originally discovered as a hotspot for adaptive mutations through serial passage experiments of R. solanacearum on host plants (Guidot et al., 2014; Capela et al., 2017). We investigated whether spontaneous

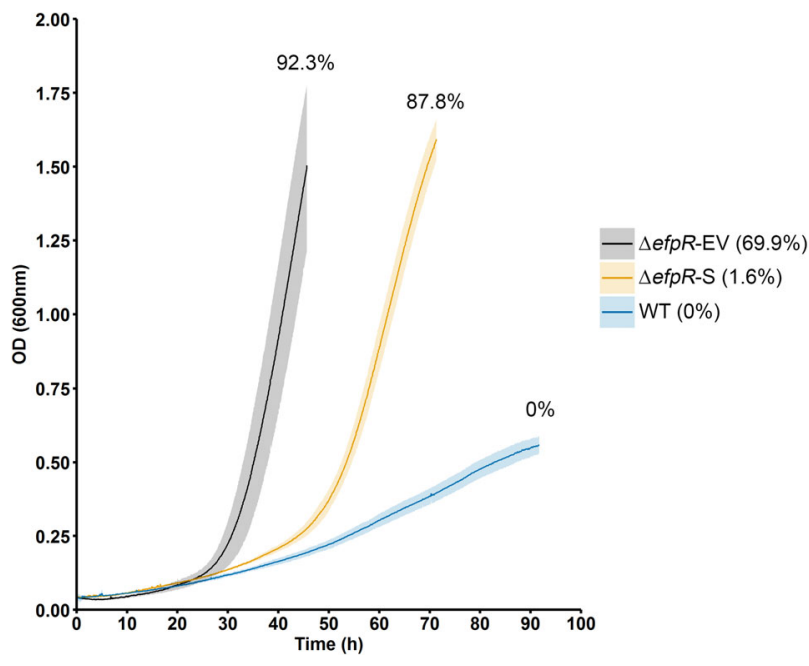

Fig. 7. Growth dynamic of $\Delta$ efpR-EV and -S types in vitro. Bacterial cultures were grown at $28^{\circ} \mathrm{C}$ in minimal medium supplemented with L-proline $(20 \mathrm{mM})$, starting from a single colony of the $\mathrm{EV}(\Delta \mathrm{efp} R)$ or $S$ types (WT and $\Delta$ efpR). The number in brackets represents the percentage of EV type CFU in the inoculum, whereas the percentage of EV type CFU at the end of the exponential growth phase is indicated above each growth curve. The dark lines represent the mean of three independent biological repeats and standard deviation is represented by the shaded region. Growth was monitored in microplates. Measurements were stopped at the end of the exponential growth phase.

mutations in efp $R$ could also be identified when bacteria are grown ex planta. We found that spontaneous mutations in efp $R$ occurred when $R$. solanacearum was maintained as a static culture in complete medium for 14 days. To ease the identification of such mutants, we used the GMl1000 strain transformed with a $\mathrm{P}_{\mathrm{RSc} 3148}$-GFP construct because expression of $R S c 3148$ is repressed by efp $R$ (Capela et al., 2017). The variants were then identified as fluorescent colonies and the efp $R$ locus was sequenced to characterize the corresponding mutations. From two independent experiments, we identified 14 clones with a SNP in the efpR gene, corresponding to three distinct mutations. Two of them are non-synonymous mutations within the efp $R$ coding sequence which, interestingly, were also detected after the in planta evolution experiment (same codon but different base substitution) (Fig. 8A). The third mutation occurred in the efp $R$ promoter region, into the predicted -35 control element (Fig. 8A).

The observation that efp $R$ variants arose in planta and ex planta and that both efp $R$ and efpH are highly conserved in all $R$. solanacearum strains sequenced to date, prompted us to examine whether phenotypic heterogeneity could be a general feature of the $R$. solanacearum species complex (RSSC). We generated efp $R$ deletion mutants in two phylogenetically distant strains: the Molk2 strain ( $R$. solanacearum, phylotype IIB) and the Psi07 strain (R. syzygii subsp. indonesiensis, phylotype IV). Both Molk2 and Psi07 $\Delta$ efpR strains were phenotypically heterogeneous, with white fluidal colonies (S type) and dark red colonies (EV type) (Fig. 8B), similarly to what 


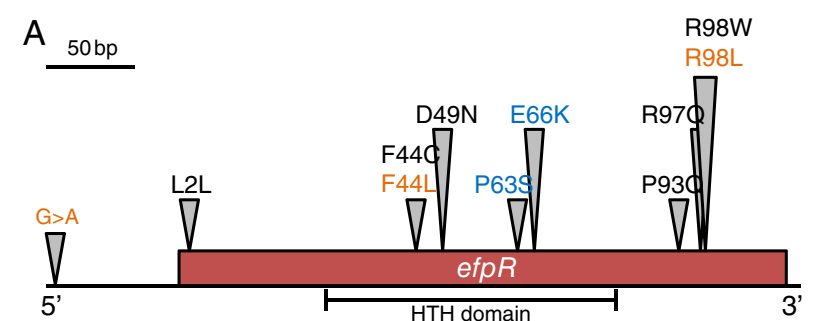

B
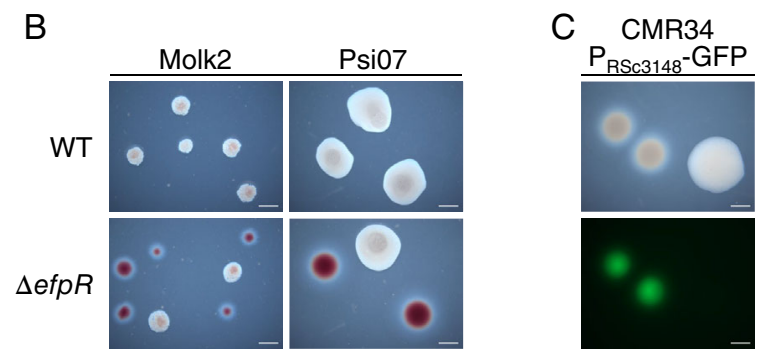

Fig. 8. The efp $R$ gene is a hotspot for adaptive mutations, which generate phenotypic heterogeneity and lead to the emergence of EV type subpopulations among strains of the $R$. solanacearum species complex. (A) Schematic representation of the various mutations identified in efp $R$ in three independent studies: in black, six mutations improving fitness of clones evolved in plant stems (Guidot et al., 2014), in blue, two mutations improving plant infection (Capela et al., 2017), and in orange, three mutations conferring fitness advantage in a static environment (this study). The region encoding the predicted helix-turn-helix $(\mathrm{HTH})$ domain in EfpR is indicated. (B) Deletion of efpR in strains Molk2 and Psi07, taxonomically distant from GMl1000, also generates phenotypic heterogeneity. (C) Expression of the RSc3148 promoter is specifically induced in EV-type colonies in strain CMR34. Bacteria were grown on complete medium agar plates for $52 \mathrm{~h}(\mathrm{~B})$ or on minimal medium agar plates for $96 \mathrm{~h}(\mathrm{C})$. Scale bar $=2 \mathrm{~mm}$.

was observed with strain GMl1000 (R. pseudosolanacearum, phylotype I).

The conservation of the efpR-mediated phenotypic heterogeneity phenomenon in phylogenetically distant strains suggested that EV type variants could be detected among natural RSSC isolates. We screened a collection of 41 strains of the $R$. solanacearum species complex isolated worldwide and representative of the taxonomic diversity of this species complex (Prior et al., 2016) (Table 1). We identified one $R$. solanacearum strain (CMR34, phylotype IIB) generating phenotypic heterogeneity stably over several subcultures on plate. This strain was transformed with the $P_{\text {RSc3148 }}$-GFP construct to determine whether the observed heterogeneity was efpR-dependent. Similarly to the GMI1000 strain, we observed EV colonies strongly expressing the reporter while it was not active in the $\mathrm{S}$ colonies (Fig. $8 \mathrm{C}$ ). These results suggest that the efpR-dependent phenotypic switch can occur among natural isolates.

\section{Discussion}

In this study, we demonstrated that loss of function mutations in the global regulator EfpR generate phenotypic heterogeneity in bacterial isogenic population of $R$. solanacearum. Indeed, efp $R$ mutants always produced two types of colonies arising from a single clone, the type $S$ ('smooth', similar to the WT) and the type EV ('efpR variant'). Both cell types have distinct phenotypic marks, highlighting that the S/EV switch phenomenon directly affects virulence and also drastically modifies bacterial cell physiology, especially by generating a form of metabolic heterogeneity in the population (Rosenthal et al., 2018; Şimşek and Kim, 2018). The efpR-dependent molecular mechanism leading to the emergence of EV type cells remains to be discovered. Recently, downregulation of efp $R$ was reported to be associated with three genes of unknown function, RSc3146-3148, two of which encoding proteins likely associated with the membrane (Capela et al., 2017), and which are presumably associated with the S/EV transition. Our study shows that the neighbouring gene, efpH (RSc3149), plays a key role in this phenotypic switch. EfpH is a homologue of EfpR (79\% identity), which also most probably operates as a transcriptional regulator. The efpH gene is not expressed in the WT strain; however, in the $\Delta$ efp $R$ strain, efpH is one of the highest upregulated gene (Perrier et al., 2016). Deletion of efpH did not generate phenotypic heterogeneity in the WT background, only forming $S$ type colonies. On the other hand, the $\Delta$ efpR- $\Delta$ efpH double mutant also did not generate phenotypic heterogeneity but only formed EV type colonies. Both efp $R$ and efpH are conserved in all the strains from the $R$. solanacearum species complex sequenced to date.

In a previous study, we showed that the efp $R$ mutant had slightly reduced virulence when inoculated on tomato plants through soil drenching (Perrier et al., 2016). Although the transcriptomic profiles of the $\Delta$ efp $R$ and $\Delta$ efpR- $\Delta$ efpH mutants were mostly similar, the strength of the signal was stronger in the double mutant (more severe downregulation of the exopolysaccharide biosynthesis gene cluster, for example). This probably explains why the $\Delta$ efpR- $\Delta$ efpH double mutant (EV type) was strongly altered in pathogenicity on tomato plants compared to the WT (S type), while pathogenicity of the $\Delta$ efp $R$ single mutant (EV $+\mathrm{S}$ types) was only mildly reduced. This suggested that the reduced disease severity on tomato plants observed for the $\Delta$ efp $R$ single mutant results from the presence of EV cells in the population.

The finding that the $\Delta \operatorname{efp} R-\Delta \operatorname{efpH}$ strain was stably 'locked' into the EV morphotype opened the way to test experimentally what is the adaptive gain for $R$. solanacearum to generate phenotypic heterogeneity. We first compared the individual fitness of EV and S cell types in different environments: the tomato plant stem, complete medium and minimal medium supplemented with different carbon sources. We found that the $S$ type had better growth ability than the EV type into plant stem, whereas the EV type had better growth ability into minimal medium. In complete medium, the EV and S types had the same fitness. These results could support the hypothesis of a bet-hedging 
Table 1. List of the WT strains of the Ralstonia solanacearum species complex investigated in this study and observation of phenotypic heterogeneity on complete BG medium.

\begin{tabular}{|c|c|c|c|c|}
\hline Strain & Phylotype & Sequevar & Plant origin & Phenotypic heterogeneity \\
\hline GMI1000 & 1 & 18 & Solanum lycopersicum & - \\
\hline TNT82A & I & 14 & Solanum Iycopersicum & - \\
\hline ACH92 & I & 116 & Zingiber officinale & - \\
\hline RUN0305 & 1 & 18 & Solanum lycopersicum & - \\
\hline TD1301 & 1 & 31 & Solanum melongena & - \\
\hline CIV23 & I & 31 & Solanum melongena & - \\
\hline RUN5501 & 1 & 31 & Solanum lycopersicum & - \\
\hline RUN3568 & 1 & 31 & Solanum lycopersicum & - \\
\hline RUN5536 & 1 & 18 & Solanum Iycopersicum & - \\
\hline $02-167$ & 1 & 18 & Solanum lycopersicum & - \\
\hline RUN3573 & I & 14 & Solanum lycopersicum & - \\
\hline RUN5445 & I & 33 & Capsicum frutescens & - \\
\hline E'4 T2 & 1 & 47 & Solanum lycopersicum & - \\
\hline RUN5135 & i & 18 & Cyphostemma mappia & - \\
\hline T1-UY & IIA & 50 & Solanum lycopersicum & - \\
\hline CFBP7054 & $\| A$ & 52 & Solanum lycopersicum & - \\
\hline CFBP2958 & IIA & 39 & Solanum lycopersicum & - \\
\hline CFBP6779 & IIA & 38 & Canna indica & - \\
\hline CMR90 & IIA & 35 & Solanum lycopersicum & - \\
\hline Molk2 & IIB & 3 & Banana & - \\
\hline CMR34 & IIB & 1 & Solanum lycopersicum & + \\
\hline CIP417 & IIB & 3 & Peanut & - \\
\hline IBSBF2001 & IIB & 25 & Solanum lycopersicum & - \\
\hline RUN3733 & III & 19 & Solanum lycopersicum & - \\
\hline CMR15 & III & 29 & Solanum lycopersicum & - \\
\hline DGBBC1222 & III & 42 & Solanum tuberosum & - \\
\hline JT525 & III & 19 & Pelargonium asperum & - \\
\hline DGBBC1125 & III & 43 & Solanum tuberosum & - \\
\hline CMR66 & III & 49 & Solanum scabrum & - \\
\hline PSI07 & IV & 10 & Solanum lycopersicum & - \\
\hline RUN1308 & IV & 10 & Solanum lycopersicum & - \\
\hline $\mathrm{ACH} 732$ & IV & 11 & Solanum lycopersicum & - \\
\hline MAFF301558 & IV & 8 & Solanum tuberosum & - \\
\hline R229 & IV & 10 & Banana & - \\
\hline UQRS91 & IV & 10 & Solanum lycopersicum & - \\
\hline RUN1309 & IV & 10 & Solanum lycopersicum & - \\
\hline UQRS280 & IV & 10 & Solanum lycopersicum & - \\
\hline UQRS290 & IV & 10 & Solanum lycopersicum & - \\
\hline PSI36 & IV & 10 & Solanum lycopersicum & - \\
\hline UW522 & IV & 11 & Clove & - \\
\hline RUN4606 & IV & 10 & Solanum tuberosum & - \\
\hline
\end{tabular}

strategy for $R$. solanacearum isogenic populations generating phenotypic heterogeneity with one part of the population expressing the $\mathrm{S}$ phenotype (virulent) better adapted to the plant environment while the other part expressing the EV phenotype is potentially better adapted to other environments such as minimal medium conditions. This also suggests that the generation of phenotypic heterogeneity in $R$. solanacearum population is certainly an advantageous strategy during the pathogen's lifecycle, which includes diversified environments (host plants, asymptomatic weeds, soil, watercourses, etc.).

In order to better understand the interaction between EV and $S$ type cells, we also compared their fitness when coinoculated in a same environment. Results indicated that the EV type is significantly more fit when co-inoculated with the $S$ type than alone in planta. We demonstrated that the reactivation of EPS production in the EV type improves its virulence, thus suggesting that the EV type could benefit from the EPS produced by the $S$ type to improve its colonization and virulence in planta. However, our results also demonstrated that the $S$ type is less fit in planta when coinoculated with the EV type than alone. As the EV type has expanded metabolic capacities, we hypothesized that the EV type consumes the nutrients and divide faster, to the detriment of the $S$ type, and in addition, does not pay the cost for EPS production and virulence (Peyraud et al., 2016).

The efp $R$ gene was originally discovered by characterizing mutations which appeared spontaneously in this gene after 300 generations of experimental evolution of $R$. solanacearum in the stem of different plants (Guidot et al., 2014; Perrier et al., 2016). Mutations in efpR were also reported to occur in strain GMI 1000 when used to be experimentally evolved into a legume symbiont (Capela et al., 2017). In this study, we also found that mutations in efpR 
can be easily selected in stressing environments such as in vitro static cultures. This observation suggested that efp $R$ variants might also exist in nature. A first argument supporting this view came from the careful analysis of a collection of WT strains, among which one of them presented the typical features of EV-S type subpopulations. Experimental testing of this hypothesis will require a specific sampling of the EV and $S$ type colonies in the various natural environments inhabited by $R$. solanacearum.

This study highlights the potential significance of generating phenotypic heterogeneity in $R$. solanacearum isogenic populations as an adaptive strategy to changing environments, mediated by spontaneous mutations in the global regulator EfpR. Spontaneous mutations in an other global regulator, PhcA, were also reported to generate avirulent but fast-growing phenotypic variants (Peyraud et al., 2016; Khokhani et al., 2017; Perrier et al., 2018). However, phcA mutations generate a single phenotype and reversion of phcA mutants to the WT form certainly occurs at a very low frequency considering the nature of genetic alterations reported (Poussier et al., 2003). Conversely, efpR mutations ensure that a mix of $E V$ and $S$ types are produced, thus maintaining a proportion of virulent population required to ensure efficient plant infection and eventually the ecological success of this pathogen. The impact of this phenotypic heterogeneity on pathogenicity opens new perspectives to tackle population dynamics underlying the bacterial wilt disease. Future sampling paying attention to EV type variants differing from the $S$ type should provide a first estimate on the frequency of the efpR-mediated heterogeneity phenomenon in natural populations.

\section{Experimental procedures}

Bacterial strains, plant material and growth conditions

Ralstonia solanacearum strains used in this study are described in Table 1 and Supporting Information Table S3. Strains were grown in complete medium or in minimal medium at $28^{\circ} \mathrm{C}$ (Plener et al., 2010). The $\mathrm{pH}$ of the minimal medium was adjusted to 6.5 with $\mathrm{KOH}$. For agar plates, the complete medium was supplemented with D-glucose $(5 \mathrm{~g}$ $\left.\mathrm{I}^{-1}\right)$ and triphenyl tetrazolium chloride $\left(0.05 \mathrm{~g} \mathrm{I}^{-1}\right)$ and the minimal medium with sucrose $\left(5 \mathrm{gl}^{-1}\right)$ when necessary. The minimal medium was supplemented with carbon sources at $20 \mathrm{mM}$ final concentration. When needed, antibiotics were added to the media at the following final concentrations $\left(\mathrm{mg} \mathrm{l}^{-1}\right)$ : spectinomycin, 40; gentamicin, 10 kanamycin, 50. Four-week-old tomato plants (Solanum lycopersicum var. SuperMarmande) were used for the inoculations. Plant experiments were conducted in a growth chamber under the following conditions: $75 \%$ humidity, $12 \mathrm{~h}$ light $28^{\circ} \mathrm{C}, 12 \mathrm{~h}$ darkness $27^{\circ} \mathrm{C}$.

\section{Transcriptome analysis}

Total RNA was extracted from the WT GMI1000 strain, the $\Delta$ efpR mutant (GRS704) and the $\Delta$ efpR- $\Delta$ efpH double mutant (GRS908) growing in complete medium at comparable cell densities $\left(O D_{600 n m}=0.5\right)$. Three biologically independent experiments were conducted for each strain. Total RNA extraction and ribosomal RNA depletion were performed as previously described (Perrier et al., 2016; 2018).

Oriented paired-end RNA sequencing $(2 \times 125 \mathrm{bp})$ was carried out by Fasteris (Fasteris SA, Plan-les-Ouates, Switzerland), using an Illumina HiSeq 2500 instrument and the procedures recommended by Illumina, with adaptors and amplification primers designed by Fasteris. The size of selected inserts was 150-250 bp.

Mapping of the reads and statistical analysis were conducted as previously described (Perrier et al., 2018). Between 0.69 and 3.86 millions of mapped read-pairs in coding sequences were obtained. Mapped reads were imported into the R environment. The package HTSFilter was used to eliminate very low expressed genes from the analysis. A total of 4930 genes out the 5307 predicted genes were thus kept in. The $R$ package DESeq2 was used to normalize and complete the differential analysis by conducting the built-in Wald test (Anders and Huber, 2010). The $p$ values thus obtained were adjusted for multiple comparisons using the falsediscovery rate (FDR) method (Strimmer, 2008). Genes with a FDR-adjusted $p<0.05$ and an absolute Fold Change I $\mathrm{FCl}>2$ between strains were taken into consideration.

\section{Construction of mutant strains and genetic constructs}

Disruption of the efpH gene was performed by creating an unmarked gene deletion using sacB-mediated counter selection (Schäfer et al., 1994). Briefly, upstream and downstream regions of efpH were PCR amplified using the primer pairs oAP35-36 and oAP37-38, respectively, and cloned into the EcoRl-HindIII-digested pk18mobsacb to generate pK18-efpH (Supporting Information Table S3). The circular pK18-efpH plasmid was inserted in the WT and $\Delta$ efpR strains by natural transformation (Perrier et al., 2016). Kanamycin-resistant and sucrose-sensitive recombinant clones were first selected to launch an overnight culture in complete medium. In a second step, kanamycin-sensitive and sucrose-resistant clones were screened by PCR using the primer pair oAP33-34 to identify a $\Delta$ efpH recombinant.

To complement the $\Delta$ efpR- $\Delta$ efpH strain, a 1044 bp fragment was PCR-amplified using the primer pair oAP41-99 and cloned into the Acc65I-Bg/ll-digested pNP329 vector to generate pNP329-efpH (Supporting Information Table S3). The pNP329-efpH plasmid was linearized with Scal before natural transformation.

To construct the strains expressing constitutively the $x p s R$ gene, the pNP329- $\mathrm{P}_{\text {tac }}-x p s R$ plasmid was constructed. A 
$1051 \mathrm{bp}$ fragment was PCR-amplified using the primers pair OAP171-176 and cloned into the Ndel-Bglll-digested pNP329- $P_{\text {tac }}$ (Supporting Information Table S3). The pNP $329-P_{\text {tac }}-x p s R$ plasmid was linearized with $\mathrm{Nhel}$ before natural transformation. Transformants were selected on gentamicin selective medium and checked by PCR using the primer pair oNP611-612.

Nucleotidic sequences of the primers used in this study are given in the Supporting Information Table S4. Construction of fluorescent strains using mCherry or GFP reporter constructs (Cruz et al., 2013) for expression studies is detailed in the Supporting Information Material and Methods file 1.

\section{Virulence tests}

Sixteen tomato plants were inoculated by soil drenching with $500 \mathrm{ml}$ of a $5 \times 10^{7} \mathrm{CFU} \mathrm{m^{-1 }}$ bacterial suspension. Plant symptoms were scored daily using a disease-index scale ranging from 0 (no symptoms) to 4 (complete wilting) as previously described (Poueymiro et al., 2009). Virulence assays were repeated three times for each strain. Disease scoring was transformed into binary data, with a disease index below 3 corresponding to 0 and a disease index equal to or higher than 3 corresponding to 1 . This transformation was performed in order to construct survival curves and to apply the survival analysis statistical protocols (Machin et al., 2006). Gehan-Breslow-Wilcoxon test was used to determine significant difference between strains using the Prism software (Prism, GraphPad, San Diego).

\section{In planta competition assay}

Two millilitres of each strains carrying different reporter fusions (GFPuv and mCherry) and grown overnight were centrifuged for $2 \mathrm{~min}$ at $17000 \mathrm{~g}$. The pellet was resuspended in $2 \mathrm{ml}$ of sterile water, and then diluted to $10^{8} \mathrm{CFU} \mathrm{ml}^{-1}$, mixed in equal proportion and diluted again to $10^{6} \mathrm{CFU} \mathrm{ml}{ }^{-1}$. Competition was performed on 4-weekold tomato inoculated by stem injection with $10 \mu \mathrm{l}$ of the mixed inoculum, $1 \mathrm{~cm}$ above the cotyledons. After 3 days, $1 \mathrm{~cm}$ of stem was harvested $2.5 \mathrm{~cm}$ above the cotyledons, placed in a $2 \mathrm{ml}$ centrifuge tube containing glass beads ( $2 \mathrm{~mm}$ diameter) and grinded during $1 \mathrm{~min}$ at $30 \mathrm{~Hz}$ with a mixer mill (MM 400, Retsch, Germany). Extracted bacteria were resuspended in $1 \mathrm{ml}$ of sterile water, serial-diluted and plated on complete medium using an automatic spiral plater (easySpiral, Interscience, France). Green and red colonies were visualized and enumerated using a fluorescence stereo zoom microscope (Axio Zoom.V16, ZEISS, Germany). Three independent biological repeats were performed with 10-18 plants per repeats per conditions. Kruskal-Wallis and Dunn's multiple comparison tests were used to establish statistical groups using the Prism software (Prism, GraphPad, San Diego).

\section{In vitro competition assay}

Bacteria were grown as described previously, except that the bacterial pellet was resuspended in $2 \mathrm{ml}$ of either complete or minimal medium supplemented with the tested carbon source (20 mM final concentration). Bacterial cultures were diluted to $5 \times 10^{7} \mathrm{CFU} \mathrm{ml}{ }^{-1}$, mixed in equal proportion and diluted again to $5 \times 10^{5} \mathrm{CFU} \mathrm{ml}^{-1}$ in a final volume of $15 \mathrm{ml}$. This volume was split in three independent test tubes of $5 \mathrm{ml}$ and incubated at $28^{\circ} \mathrm{C}$ under shaking at $180 \mathrm{rpm}$ until the culture reach $\mathrm{OD}_{600 \mathrm{~nm}}>1$. Plating of bacteria and fluorescence detection was performed as described previously. Two independent biological repeats, each comprising three technical repeats, were performed.

\section{Monitoring in vitro growth}

Bacteria were grown overnight in complete liquid medium and plated in order to have isolated colonies. Single colonies (EV or S) were resuspended in minimal medium supplemented with L-Proline $(20 \mathrm{mM})$ and diluted to $5 \times 10^{7}$ $\mathrm{CFU} \mathrm{ml}{ }^{-1}$. An aliquot of $100 \mu \mathrm{l}$ was used to determine the initial proportion of EV and S CFU and $200 \mu \mathrm{l}$ was used as inoculum. Bacterial growth was monitored using a microplate spectrophotometer (FLUOstar Omega, BMG Labtech, Germany) during $91 \mathrm{~h}$ at $28^{\circ} \mathrm{C}$ under shaking at $700 \mathrm{rpm}$ using a linear shaking mode. At the end of the exponential growth phase, $100 \mu \mathrm{l}$ was harvested for bacterial enumeration and fluorescence detection. Three independent biological repeats were performed.

\section{In vitro static culture experiment}

Glass haemolysis tubes were filled with $5 \mathrm{ml}$ of complete medium and inoculated with a single colony of strain GMl1000 bearing the $P_{\mathrm{RSc} 3148}$-GFP construct. After 14 days at $28^{\circ} \mathrm{C}$ without agitation, $10 \mu$ of the liquid surface was recovered, serial-diluted and plated on complete medium in order to have isolated colonies. Green fluorescent colonies were visualized as described above. The efp $R$ locus of each purified clone was PCR-amplified with the primers oCBM2872-oAP77 and sequenced using primers oAP76 and oCBM2440 (Supporting Information Table S4). Five independent biological repeats were performed.

\section{Acknowledgements}

We thank Jean-Baptiste Ferdy, Alain Givaudan, Laurent Noël, Rémi Peyraud and Philippe Remigi for support and advices during the course of this work. Delphine Capela, Nemo Peeters and Laurent Deslandes for supplying 
plasmids and/or primers. We also thank Patrick Barberis, Claire Benezech and Maëlys Puyo for technical assistance, and the Plateforme Imagerie from the Institut Fédératif de Recherche 3450. We acknowledge Richard Berthomé for the $R$. solanacearum strain collection used in this work, and Benoit Facon and Fabienne Vailleau for the observation of the colony morphotypes on plate of the CMR34 strain in La Réunion.

\section{References}

Acar, M., Mettetal, J.T., and van Oudenaarden, A. (2008) Stochastic switching as a survival strategy in fluctuating environments. Nat Genet 40: 471-475.

Ackermann, M. (2015) A functional perspective on phenotypic heterogeneity in microorganisms. Nat Rev Microbiol 13: 497-508.

Anders, S., and Huber, W. (2010) Differential expression analysis for sequence count data. Genome Biol 11: R106.

Arnoldini, M., Vizcarra, I.A., Peña-Miller, R., Stocker, N., Diard, M., Vogel, V., et al. (2014) Bistable expression of virulence genes in salmonella leads to the formation of an antibiotic-tolerant subpopulation. PLoS Biol 12: e1001928.

Balaban, N.Q., Merrin, J., Chait, R., Kowalik, L., and Leibler, S. (2004) Bacterial persistence as a phenotypic switch. Science 305: 1622-1625.

Capela, D., Marchetti, M., Clérissi, C., Perrier, A., Guetta, D., Gris, C., et al. (2017) Recruitment of a lineage-specific virulence regulatory pathway promotes intracellular infection by a plant pathogen experimentally evolved into a legume symbiont. Mol Biol Evol 34: 2503-2521.

Casadesús, J., and Low, D.A. (2013) Programmed heterogeneity: epigenetic mechanisms in bacteria. $J$ Biol Chem 288: 13929-13935.

Chai, Y., Chu, F., Kolter, R., and Losick, R. (2008) Bistability and biofilm formation in Bacillus subtilis. Mol Microbiol 67: 254-263.

Chin, C.Y., Tipton, K.A., Farokhyfar, M., Burd, E.M., Weiss, D.S., and Rather, P.N. (2018) A high-frequency phenotypic switch links bacterial virulence and environmental survival in Acinetobacter baumannii. Nat Microbiol 3: 563-569.

Cruz, A.P.Z., Ferreira, V., Pianzzola, M.J., Siri, M.I., Coll, N. S., and Valls, M. (2013) A novel, sensitive method to evaluate potato Germplasm for bacterial wilt resistance using a luminescent Ralstonia solanacearum reporter strain. Mol Plant Microbe Interact 27: 277-285.

Diard, M., Garcia, V., Maier, L., Remus-Emsermann, M.N.P., Regoes, R.R., Ackermann, M., and Hardt, W.-D. (2013) Stabilization of cooperative virulence by the expression of an avirulent phenotype. Nature 494: 353-356.

Fraser, D., and Kaern, M. (2009) A chance at survival: gene expression noise and phenotypic diversification strategies. Mol Microbiol 71: 1333-1340.

Genin, S., and Denny, T.P. (2012) Pathogenomics of the Ralstonia solanacearum species complex. Annu Rev Phytopathol 50: 67-89.

Guidot, A., Jiang, W., Ferdy, J.-B., Thébaud, C., Barberis, P., Gouzy, J., and Genin, S. (2014) Multihost experimental evolution of the pathogen Ralstonia solanacearum unveils genes involved in adaptation to plants. Mol Biol Evol 31: 2913-2928.

Hallet, B. (2001) Playing Dr Jekyll and Mr Hyde: combined mechanisms of phase variation in bacteria. Curr Opin Microbiol 4: 570-581.

Harrison, F. (2013) Dynamic social behaviour in a bacterium: Pseudomonas aeruginosa partially compensates for siderophore loss to cheats. J Evol Biol 26: 1370-1378.

Huang, J., Carney, B.F., Denny, T.P., Weissinger, A.K., and Schell, M.A. (1995) A complex network regulates expression of eps and other virulence genes of pseudomonas solanacearum. J Bacteriol 177: 1259-1267.

Khokhani, D., Lowe-Power, T.M., Tran, T.M., and Allen, C. (2017) A single regulator mediates strategic switching between attachment/spread and growth/virulence in the plant pathogen Ralstonia solanacearum. MBio 8: e00895-e00817.

Machin, D., Cheung, Y.B., and Parmar, M. (2006) Survival Analysis: A Practical Approach, 2nd edn. Oxford, UK: Wiley, pp, 278.

Mansfield, J., Genin, S., Magori, S., Citovsky, V., Sriariyanum, M., Ronald, P., et al. (2012) Top 10 plant pathogenic bacteria in molecular plant pathology. Mol Plant Pathol 13: 614-629.

Mouammine, A., Pages, S., Lanois, A., Gaudriault, S., Jubelin, G., Bonabaud, M., et al. (2017) An antimicrobial peptide-resistant minor subpopulation of Photorhabdus luminescens is responsible for virulence. Sci Rep 7: 43670.

Peeters, N., Guidot, A., Vailleau, F., and Valls, M. (2013) Ralstonia solanacearum, a widespread bacterial plant pathogen in the post-genomic era. Mol Plant Pathol 14: 651-662.

Perrier, A., Barlet, X., Peyraud, R., Rengel, D., Guidot, A., and Genin, S. (2018) Comparative transcriptomic studies identify specific expression patterns of virulence factors under the control of the master regulator PhcA in the Ralstonia solanacearum species complex. Microb Pathog 116: 273-278.

Perrier, A., Peyraud, R., Rengel, D., Barlet, X., Lucasson, E., Gouzy, J., et al. (2016) Enhanced in planta fitness through adaptive mutations in EfpR, a dual regulator of virulence and metabolic functions in the plant pathogen Ralstonia solanacearum. PLoS Pathog 12: e1006044.

Peyraud, R., Cottret, L., Marmiesse, L., and Genin, S. (2018) Control of primary metabolism by a virulence regulatory network promotes robustness in a plant pathogen. Nat Commun 9: 418.

Peyraud, R., Cottret, L., Marmiesse, L., Gouzy, J., and Genin, S. (2016) A resource allocation trade-off between virulence and proliferation drives metabolic versatility in the plant pathogen Ralstonia solanacearum. PLoS Pathog 12: e1005939.

Plener, L., Manfredi, P., Valls, M., and Genin, S. (2010) PrhG, a transcriptional regulator responding to growth conditions, is involved in the control of the type III secretion system regulon in Ralstonia solanacearum. J Bacteriol 192: 1011-1019.

Poueymiro, M., Cunnac, S., Barberis, P., Deslandes, L., Peeters, N., Cazale-Noel, A.-C., et al. (2009) Two type III secretion system effectors from Ralstonia solanacearum GMI1000 determine host-range specificity on tobacco. Mol Plant-Microbe Interact MPMI 22: 538-550. 
Poussier, S., Thoquet, P., Trigalet-Demery, D., Barthet, S., Meyer, D., Arlat, M., and Trigalet, A. (2003) Host plantdependent phenotypic reversion of Ralstonia solanacearum from non-pathogenic to pathogenic forms via alterations in the phcA gene. Mol Microbiol 49: 991-1003.

Prior, P., Ailloud, F., Dalsing, B.L., Remenant, B., Sanchez, B., and Allen, C. (2016) Genomic and proteomic evidence supporting the division of the plant pathogen Ralstonia solanacearum into three species. BMC Genomics 17: 90.

Rainey, P.B., Beaumont, H.J., Ferguson, G.C., Gallie, J., Kost, C., Libby, E., and Zhang, X.-X. (2011) The evolutionary emergence of stochastic phenotype switching in bacteria. Microb Cell Fact 10: S14.

Ronin, I., Katsowich, N., Rosenshine, I., and Balaban, N.Q. (2017) A long-term epigenetic memory switch controls bacterial virulence bimodality. eLife 6: e19599.

Rosenthal, A.Z., Qi, Y., Hormoz, S., Park, J., Li, S.H.-J., and Elowitz, M.B. (2018) Metabolic interactions between dynamic bacterial subpopulations. eLife 7: e33099.

Rufián, J.S., Macho, A.P., Corry, D.S., Mansfield, J.W., Ruiz-Albert, J., Arnold, D.L., and Beuzón, C.R. (2018) Confocal microscopy reveals in planta dynamic interactions between pathogenic, avirulent and non-pathogenic pseudomonas syringae strains. Mol Plant Pathol 19: 537-551.

Rufián, J.S., Sánchez-Romero, M.-A., López-Márquez, D., Macho, A.P., Mansfield, J.W., Arnold, D.L., et al. (2016) Pseudomonas syringae differentiates into phenotypically distinct subpopulations during colonization of a plant host. Environ Microbiol 18: 3593-3605.

Schäfer, A., Tauch, A., Jäger, W., Kalinowski, J., Thierbach, G., and Pühler, A. (1994) Small mobilizable multi-purpose cloning vectors derived from the Escherichia coli plasmids pK18 and pK19: selection of defined deletions in the chromosome of Corynebacterium glutamicum. Gene 145: 69-73.

Schreiber, F., Littmann, S., Lavik, G., Escrig, S., Meibom, A., Kuypers, M.M.M., and Ackermann, M. (2016) Phenotypic heterogeneity driven by nutrient limitation promotes growth in fluctuating environments. Nat Microbiol 1: 16055.

Şimşek, E., and Kim, M. (2018) The emergence of metabolic heterogeneity and diverse growth responses in isogenic bacterial cells. ISME J 12: 1199-1209.

Stewart, M.K., Cummings, L.A., Johnson, M.L., Berezow, A. B., and Cookson, B.T. (2011) Regulation of phenotypic heterogeneity permits salmonella evasion of the host caspase-1 inflammatory response. Proc Natl Acad Sci U $S$ A 108: 20742-20747.

Strassmann, J.E. (2000) Evolution: bacterial cheaters. Nature 404: 555-556.

Strimmer, K. (2008) A unified approach to false discovery rate estimation. BMC Bioinformatics 9: 303.

Tuchscherr, L., Medina, E., Hussain, M., Völker, W., Heitmann, V., Niemann, S., et al. (2011) Staphylococcus aureus phenotype switching: an effective bacterial strategy to escape host immune response and establish a chronic infection. EMBO Mol Med 3: 129-141.

Veening, J.-W., Smits, W.K., and Kuipers, O.P. (2008) Bistability, epigenetics, and bet-hedging in bacteria. Annu Rev Microbiol 62: 193-210.
Weigel, W.A., and Dersch, P. (2018) Phenotypic heterogeneity: a bacterial virulence strategy. Microbes Infect 20: 570-577.

Workentine, M.L., Sibley, C.D., Glezerson, B., Purighalla, S., Norgaard-Gron, J.C., Parkins, M.D., et al. (2013) Phenotypic heterogeneity of $P$ seudomonas aeruginosa populations in a cystic fibrosis patient. PLoS One 8: e60225.

\section{Supporting Information}

Additional Supporting Information may be found in the online version of this article at the publisher's web-site:

Appendix S1: Supplementary-Material.

Figure S1. Observation of phenotypic heterogeneity in Ralstonia solanacearum GMI1000 $\Delta$ efpR and efpR SNP mutants obtained after experimental evolution in planta. Colonies formed after $52 \mathrm{~h}$ at $28^{\circ} \mathrm{C}$ on complete medium agar plates supplemented with D-glucose and triphenyl tetrazolium chloride red are observed under a stereo-microscope. Black arrows indicate less mucoid colonies compared to the GMl1000 wild-type (WT) colonies, with a red center and layered edge. $\triangle$ efpR::efpR and $\Delta$ efpR$\Delta$ efpH::efpH are the efpR and efpH complemented strains, respectively. efp $R^{\mathrm{P9} Q \mathrm{Q}}$, efp $R^{\mathrm{L2L}}$ and efp $R^{\mathrm{D} 49 \mathrm{~N}}$ are the in planta evolved efp $R$ SNP mutants. Scale bar $=2 \mathrm{~mm}$.

Figure S2. Differential expression pattern of the fliC promoter in $\mathbf{S}$ and $\mathrm{EV}$ type colonies. Left panels show bright field images; the right panels show the same colonies under fluorescence excitation. For the $\Delta$ efp $R$ strain, one type EV (left) and one type $S$ (right) colonies are shown. Expression of the $P_{\text {flic }}$-mCherry reporter occurs only in EV type colonies. Bacteria were grown on BG agar plates for $52 \mathrm{~h}$ at $28^{\circ} \mathrm{C}$. Scale bar $=2 \mathrm{~mm}$.

Figure S3. Comparison of the fliC promoter activity in R. solanacearum GMI1000 wild-type (WT), $\Delta$ efpR and $\Delta$ efpR- $\Delta$ efpH populations after overnight growth in complete liquid medium. To visualize $R$. solanacearum cells, overnight cultures in complete liquid medium were diluted ten times and $4 \mu \mathrm{l}$ was mounted between a $1 \mathrm{~mm} 1 \%$ agarose pad on a microscope slide and a cover slip. Bacteria were imaged using a Leica TCS SP8 confocal laser microscope, with a 40x water objective and the LAS X software (Leica).

Left panels show bright field images of the 3 different strains, the middle panels show eGFP fluoresence profile of the 3 strains and the right panels show the overlay of the two first panel. In the WT strain, the fliC promoter is weakly active in all the cells while in the $\Delta$ efpR strain, the promoter is weakly active in a subpopulation (white arrows) and an other subpopulation the promoter is strongly active. In the $\Delta$ efpR- $\Delta$ efpH strain the fliC promoter is strongly active in most of the cells, a few cells per field show low activity pattern. Scale bar $=10 \mu \mathrm{m}$.

Figure S4. Impact of the initial ratio of the EV and S types on the in planta fitness of the population. Bacteria were co-injected in tomato stems at various initial ratio $(100: 0,99: 1,90: 10,50: 50,10: 90)$ and harvested 3 days post-inoculation. The strain represented is 
3152 A. Perrier et al.

indicated in bold while the co-inoculated strain is indicated in brackets. Three independent biological repeats were performed with 5 to 8 plants per repeats per conditions. Bars indicate standard deviation.

Table S1. RNAseq data for all wild-type GMI1000 strain, $\Delta$ efpR mutant (GRS704) and $\Delta$ efpR- $\Delta$ efpH double mutant (GRS908) transcripts detected in complete medium.
Table S2. Differentially expressed genes (DEG) between the WT GMl1000 strain and the $\Delta \operatorname{efp} R$ mutant or the $\Delta$ efpR- $\Delta$ efpH double mutant, or between $\Delta$ efp $R$ mutant and the $\Delta$ efpR- $\Delta$ efpH double mutant in complete medium.

Table S3. List of strains and plasmids used and constructed in this study.

Table S4. List of primers used in this study. 